\title{
Competition between herbage plants
}

\author{
C. T. DE WIT and J. P. VAN DEN BERGH \\ Institute for Biological and Chemical Research on Field Crops and Herbage, \\ Wageningen, Netherlands
}

\section{Summary}

Starting from work with annuals a model of competition between herbage plants is discussed. It is shown that their mutual interference can only be described adequately if they are grown in mixture and also in monoculture.

\section{Introduction}

To find a basis for the mutual interference in pastures, DE WIT and ENNIK (1958) studied competition experiments between annual species. Their theory was worked out in considerable detail by DE WIT $(1960,1961)$ up to the level considered suitable in the interpretation of competition experiments between perennial grassland species. In due course, however, difficulties were encountered (ENNIK, 1960; VAN DEN BERGH and DE WIT, 1960; VAN DEN BERGH and ElBERSE, 1962) related to the problem of finding a stage in herbage plants reoccurring at intervals, like the seeds of annual species.

It is shown here how and to what extent this problem can be overcome.

\section{The relative reproductive rate}

The relative reproductive rate (RRR) of two annuals $a$ and $b$ is

$$
{ }^{21} \alpha_{\mathrm{ab}}=\frac{{ }^{2} O_{\mathrm{a}} / 1 O_{\mathrm{a}}}{{ }^{2} O_{\mathrm{b}} /{ }^{1} O_{\mathrm{b}}}
$$

in which ${ }^{1} O_{\mathrm{a}}$ and ${ }^{1} O_{\mathrm{b}}$ are the amount of kernels sown and ${ }^{2} O_{\mathrm{a}}$ and ${ }^{2} O_{\mathrm{b}}$ the amount of kernels harvested. It is said (FISHER, 1930; LI, 1955; DE WIT, 1960, 1961) that both species match each other when ${ }^{21} \alpha_{\mathrm{ab}}$ is 1 and that species a gains on species $\mathrm{b}$ when it is greater than 1 , tacitly assuming that the harvested kernels are replicas of the sown ones. This assumption may be often acceptable, but is never quite correct, so that it is impossible to conclude which species gains when RRR is close to 1 .

HARLAN and MARTINI (1938) cultivated during 13 years a mixture of 10 barley varieties at different places in the United States. Five hundred seeds out of each harvest were sown to obtain plants which could be determined as to variety. Results of such experiments are represented conveniently by plotting the ratio of the kernel yields of two species on a logarithmic scale against time and connecting adjacent points. These lines are called "course lines". This is done in FIG. 1 for White Smyrna(s) and

Received for publication 12th February, 1965. 


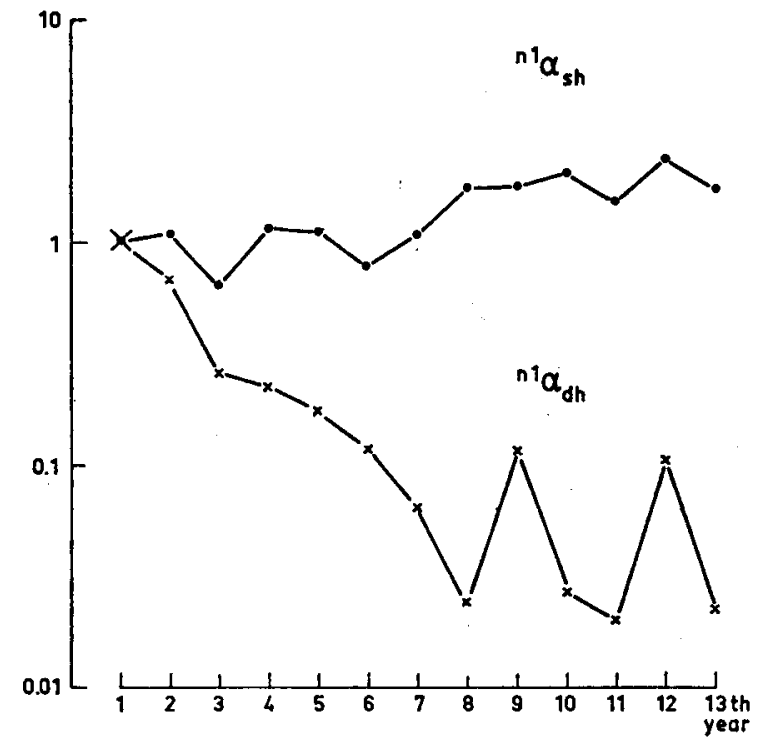

FIG. 1

Course lines for the barley varieties White Smyrna(s) and Deficiens(d), both with respect to Hannchen(h), calculated from the number of seeds in the yields of a mixture grown in Aberdeen (Idaho, U.S.A.). Data from Harlan and MARTINI (1938).

Deficiens(d) both with respect to Hannchen(h). Since the yield ratio's are 1 in the first year, these course lines also present the RRR with respect to this year.

Course lines are straight when RRR is constant with time. Horizontal lines indicate that RRR is 1 and otherwise the slope of the line is proportional to RRR. In general a broken line is found. The course of this line reflects the changes in RRR most conveniently. In this particular case White Smyrna gains slightly on Hannchen and both gain considerably on Deficiens.

The harvest of herbage plants is not resown, but the stubbles and roots are left for regrowth. Moreover, each harvest grows under different conditions and may be obtained at irregular intervals throughout the season, so that one gram of dry matter of one yield in general is not a replica of one gram of dry matter of another. Therefore it is impossible to conclude by means of herbage yields which species of the mixture gains, unless the course line is very steep or the mixture is cultivated for a very long period of time.

Instead, the number of sprouts may be counted at intervals or after each harvest. The assumption that these are replicas has been studied by means of a mixture of Lolium perenne (Lp) and Anthoxanthum odoratum (Ao) grown in pots in a climate room and harvested eight times at three weeks intervals. The number of sprouts and the herbage yields were determined at each harvest for both species. This experiment is discussed in more detail in section 5 .

Here, the size and vigour of the sprouts can be evaluated more or less by considering the herbage yields per sprout, because the pots were kept under constant conditions and harvested at regular intervals. With $L p$ the yields per sprout did not vary systematically with time, indicating that the size and vigour of the sprouts did not vary. But with Ao the yield per sprout decreased from $35 \mathrm{mg}$ in the 1 st to $4 \mathrm{mg}$ in the 8th harvest, so that the sprouts lost considerably in size and vigour.

In FIG. 2 course line I, based on the dry-matter yields, indicates that Lp gains rapidly, but course line II, based on the number of sprouts, suggests that Lp gains little. This 


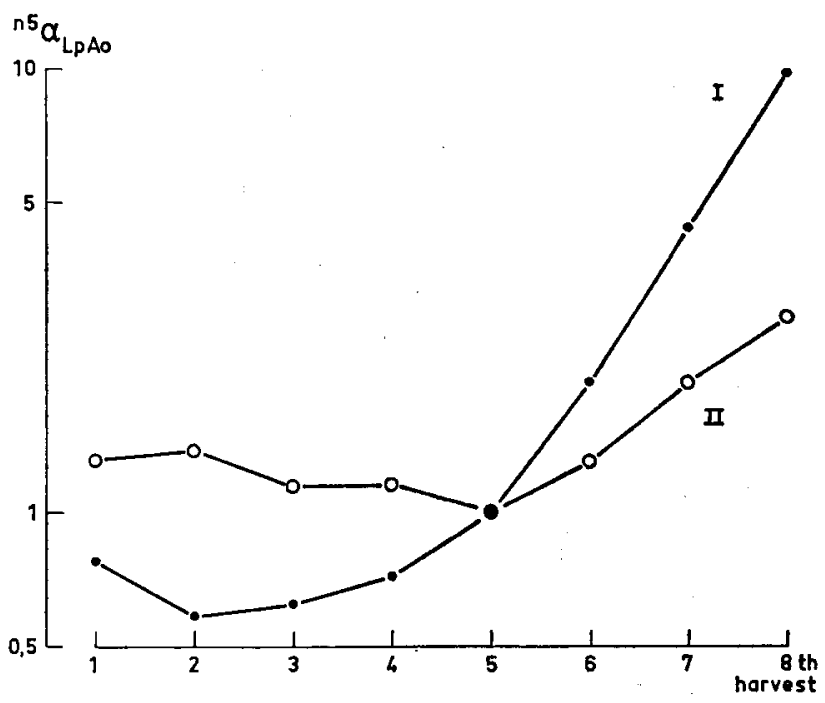

FIG. 2

The relative reproductive rates presented as course lines for Lolium (Lp) with respect to Anthoxanthum (Ao) grown in pots, calculated from the dry-matter yields (I) and the number of sprouts after cutting (II). The experimental conditions are described in section 5 .

latter course line is rather misleading because Ao had disappeared completely before the 9th harvest.

It was also attempted to determine directly the amount of material contributing to the regrowth after cutting by considering the carbohydrate reserves, regrowth in the dark and other measures. In due course, however, it was realized that it is possible to devise an unlimited number of methods to determine some relative reproductive rate, but that it is impossible to discriminate between them.

\section{The relative replacement rate}

The above mentioned difficulties may be overcome if the species are also grown in monoculture. The relative yield of a species is

$$
\mathrm{m}_{r}={ }^{\mathrm{m}} O /{ }^{\mathrm{m}} M
$$

in which ${ }^{\mathrm{m} O}$ and $\mathrm{m} M$ are the yields in mixture and in monoculture at the $\mathrm{mth}$ harvest. Now the relative replacement rate of species a with respect to species $b$ at the nth harvest with respect to the $m$ th harvest is defined as

$$
{ }^{n m} \varrho_{a b}=\frac{{ }^{n} r_{a} /{ }^{m} r_{a}}{{ }^{n} r_{b} /{ }^{m} r_{b}}
$$

The relative replacement rate of annuals may be obtained if the harvests of the species in monoculture and in mixture are resown. In that case

$$
{ }^{\mathrm{nm}} \varrho_{\mathrm{ab}}=\frac{{ }^{\mathrm{n}} O_{\mathrm{a}} /{ }^{\mathrm{m}} O_{\mathrm{a}}}{{ }^{\mathrm{n}} O_{\mathrm{b}} /{ }^{\mathrm{m}} O_{\mathrm{b}}} \times \frac{{ }^{\mathrm{m}} M_{\mathrm{a}} /{ }^{\mathrm{n}} M_{\mathrm{a}}}{{ }^{\mathrm{m}} M_{\mathrm{b}} /{ }^{\mathrm{n}} M_{\mathrm{b}}}
$$

in which $O$ and $M$ are the kernel yields. From the time onwards the yields of the monocultures do not systematically change with time, the expectation value of the double ratio of $M$ is 1 , so that in case of annuals the expectation value of the relative replacement rate equals the expectation value of the relative reproductive rate. 
Because of this equivalence both relative rates are presented by the abreviation RRR, unless it is necessary to make a distinction.

The variations in yield due to changes in growing conditions and varying lengths of the growth period are taken into account by considering the yields of the monoculture also. However, this makes only sense if the numerical value of the relative replacement rate is practically the same, whether calculated on basis of dry-matter yields, number of sprouts or any other quantity. It appears in section 5 that this is indeed the case. At first it will be shown that a basic model of competition which was developed for annuals (DE WIT, 1960, 1961) may also be applied in case of herbage plants.

\section{Mutual exclusive species}

The relative yield total of two species is equal to the sum of the relative yields, i.e. :

$$
\mathrm{RYT}=r_{\mathrm{a}}+r_{\mathrm{b}}=O_{\mathrm{a}} / M_{\mathrm{a}}+O_{\mathrm{b}} / M_{\mathrm{b}}
$$

Two species are (mutually) exclusive when the expectation value of RYT is equal to 1 . This often occurs during the period of establishment, provided the sum of the relative planting rates is 1 , or after establishment irrespective of the scheme of planting.

Combining equations (3) and (5) gives

$$
\begin{aligned}
& { }^{\mathrm{n}} r_{\mathrm{a}}=\frac{{ }^{\mathrm{nm}} \varrho_{\mathrm{ab}} \mathrm{m}_{\mathrm{a}}}{{ }^{\mathrm{n} m} \varrho_{\mathrm{ab}}{ }^{\mathrm{m}} r_{\mathrm{a}}+{ }^{\mathrm{m}} r_{\mathrm{b}}} \\
& { }^{\mathrm{n}} r_{\mathrm{b}}=\frac{{ }^{\mathrm{m}} r_{\mathrm{b}}}{{ }^{\mathrm{n} m} \varrho_{\mathrm{ab}}{ }^{\mathrm{m}} r_{\mathrm{a}}+{ }^{\mathrm{m}} r_{\mathrm{b}}}
\end{aligned}
$$

In case of annuals it was found that ${ }^{\mathrm{mm}} \varrho_{\mathrm{ab}}$ in equation 6 is a constant which depends on experimental conditions but is practically independent of the yield ratio, when the species are exclusive. This also holds for herbage plants as is illustrated in the replacement diagram of FIG. $3 a$ which concerns a competition experiment in containers with Lolium and Anthoxanthum. The curves through the observations are drawn according to the equations (6) with ${ }^{31} \varrho_{\text {LpAo }}$ equal to 3.2. FIG. $3 b$ concerns the same experiment but for the absolute yields $O=$ r. $M$ along the vertical axis. Methods to estimate $\varrho$ from a set of experimental data are discussed by DE WIT (1960).

Theoretical curves, obtained by supposing that RRR is not constant but varies linearly from 4.2 at the left side to 2.2 at the right side of the diagram and the reverse are given in FIG. $3 \mathrm{c}$ and $3 \mathrm{~d}$. The deviations from the curves in FIG. $3 a$ are so small compared to the scattering that no great error is introduced by supposing that RRR does not depend on the yield ratio if two species are mutually exclusive.

The yields of a competition experiment for eight successive harvests are given in the eight replacement diagrams of FIG. 4. The horizontal axis concerns the 5 th harvest and the position of the points along this axis are obtained as follows. It is seen in FIG. 5 that both species are exclusive, but also that RYT directly calculated from the observations is not exactly 1 due to scattering. This is adjusted by 1 . reading the yields $O$ at the intersection of the full drawn line through the observations and the dotted lines through the origin and each of the observations, 2. reading the yields $M$ at the intersections of the full drawn line and both axis and 3 . calculating the relative yields for the reference harvest (here the 5 th) from these values. 
FIg. 3. Replacement diagrams of a competition experiment with Lolium (Lp) and Anthoxanthum (Ao) with the relative plant frequency along the horizontal axis and the yields in $g$ dry matter or the relative yields of the 3rd harvest along the vertical axis. The curves are drawn according to equations (6). In $a$ and $b{ }^{31} \varrho_{\text {LPAo }}$ is equal to 3.2. In $\mathrm{c} \varrho$ varies from 4.2 at the left side to 2.2 at the right side, whereas the reverse is the case in $\mathrm{d}$.

$x$ LP AO ORYT
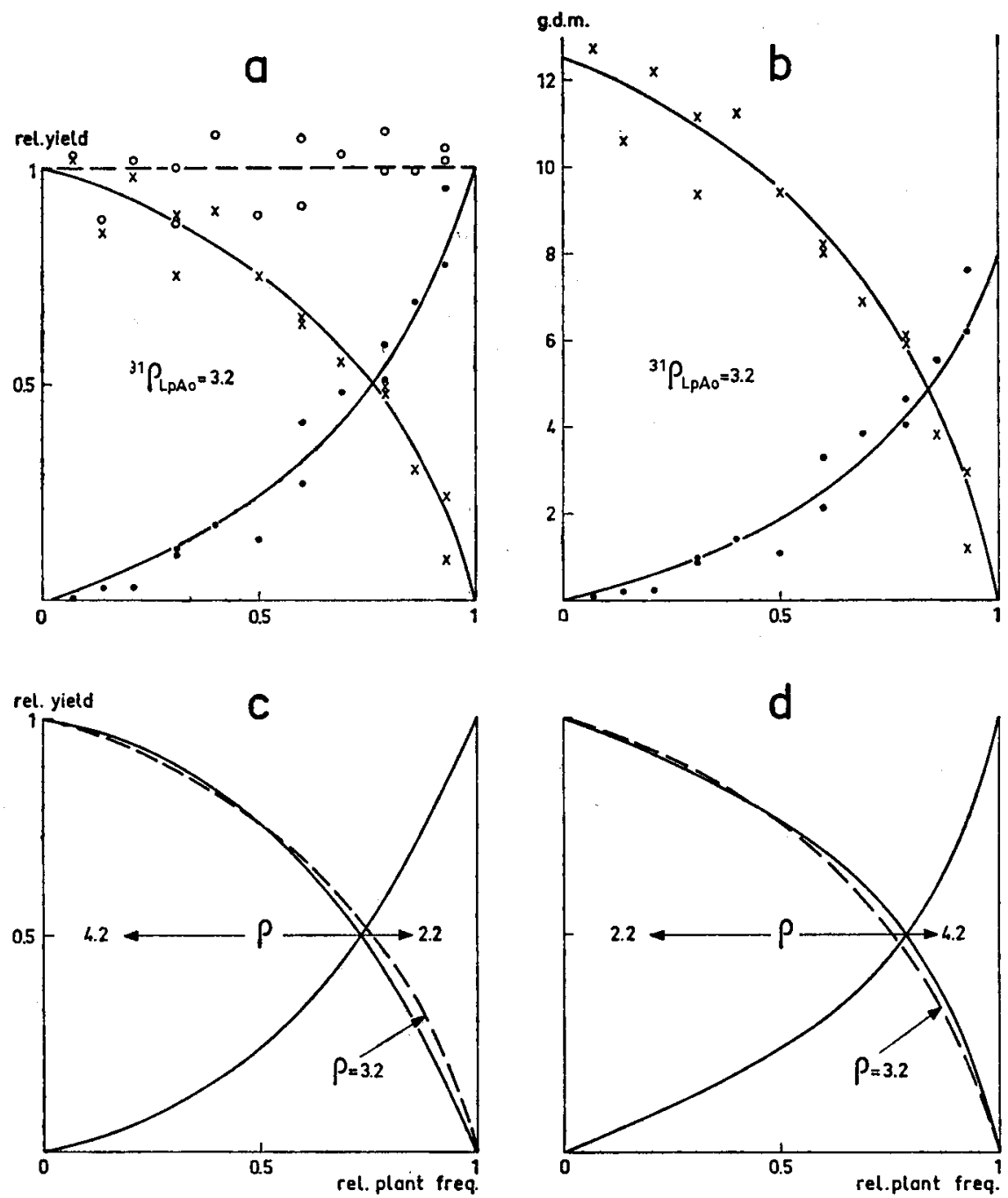

The unadjusted and adjusted relative yields are given in the TABLE and the differences between them show also in the replacement diagram for the 5th harvest in FIG. 4. The RRR's with respect to the fifth harvest are given by course line $I$ in FIG. 6 . The 
FIG. 4. Replacement diagrams based on dry-matter yields of Lolium ( $\mathrm{Lp}$ ) and Anthoxanthum (Ao) at eight successive harvests. The experimental conditions are described in section 5 .
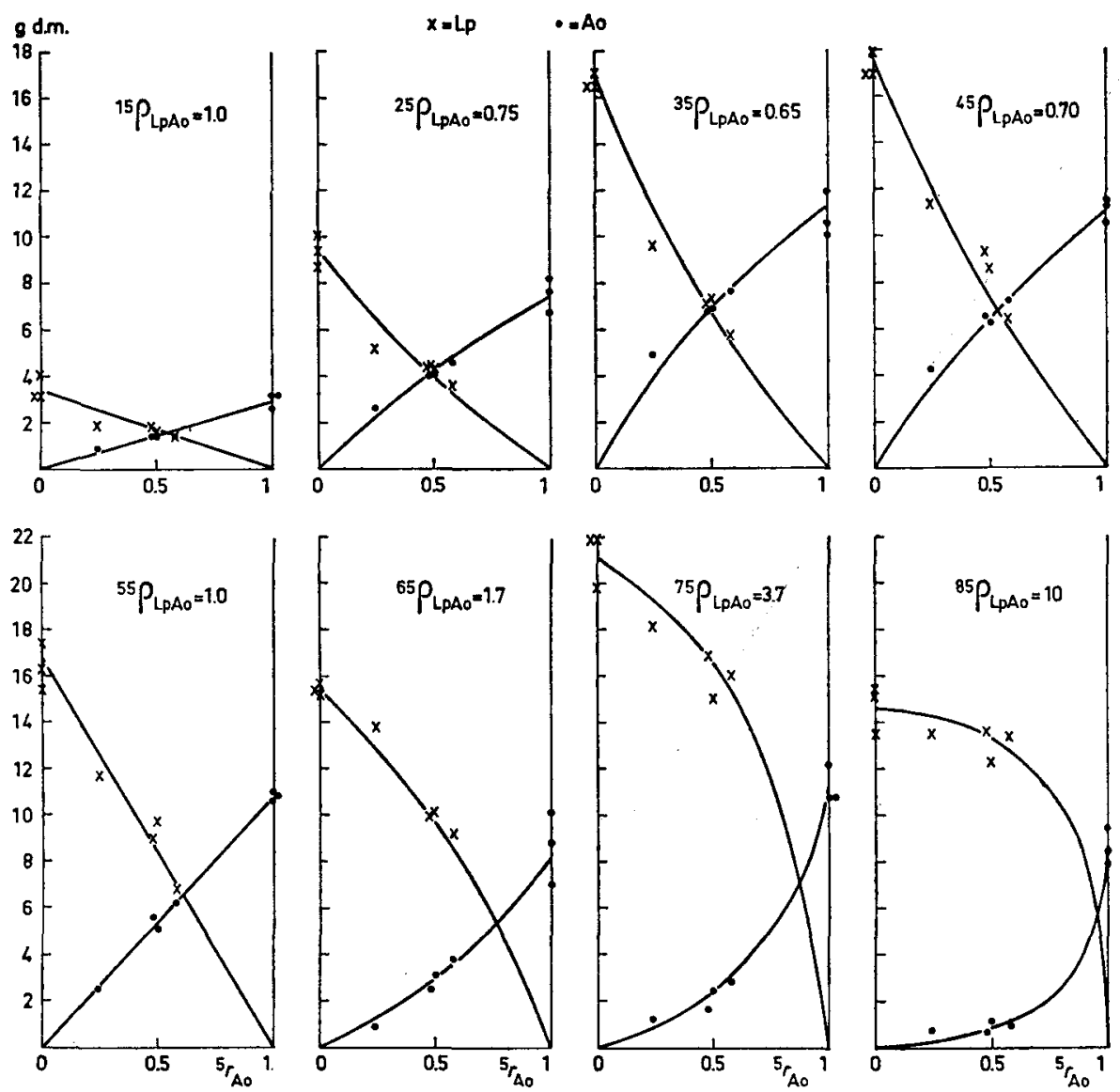

course lines for other relative yield ratio's are equidistant with this one, because RRR does not depend on the yield ratio.

Sometimes two species are not mutually exclusive, but the treatment of these cases is beyond the scope of this article.

\section{A comparison of various methods of calculating RRR}

It will be shown now that the relative replacement rate is practically independent of the method of yield determination. For this purpose pots with a diameter of $20 \mathrm{~cm}$, filled with loamy sand (Born-Zuid soil) were planted with Lolium and Anthoxanthum in monoculture and in a $1: 1$ mixture at a density of 12 sprouts. The pots were kept in a climate room at a temperature of $20^{\circ} \mathrm{C}$, a relative humidity of about 70 per cent and under HPL light at $0.07 \mathrm{cal} \mathrm{cm}^{-2} \mathrm{~min}^{-1}(400-700 \mathrm{~m} \mu)$ during 17 hours a day. The pots were well supplied with water and nutrients and clipped eight 


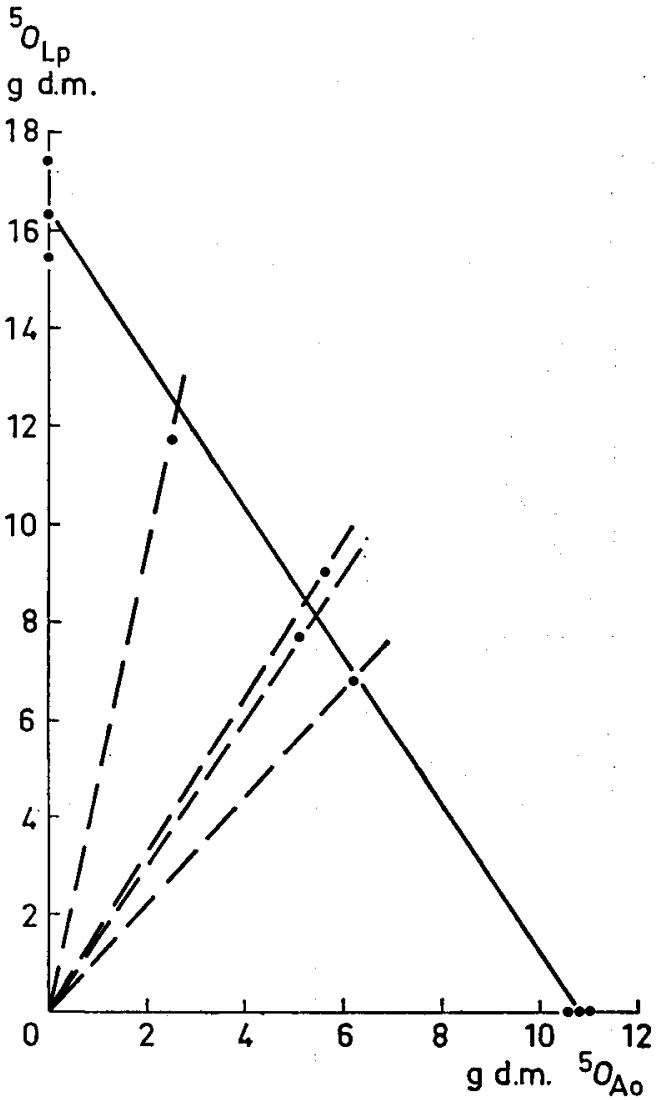

Fig. 5

Estimation of the relative yields of the reference harvest in FIG. 4 (See text).

TABLE. Adjusted and unadjusted relative yields of Lolium (Lp) and Anthoxanthum (Ao) of the 5th harvest

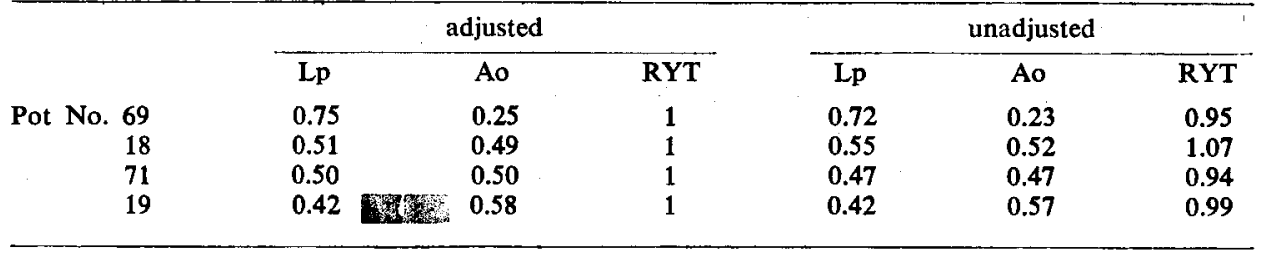

times at three weeks intervals. At each harvest the dry-matter yields and the number of sprouts of each species were determined.

The yields of the pots are given in the replacement diagrams of FIG. 4, with the 5th harvest as reference. Both species are exclusive and the RRR is given by course line I of FIG. 6. During the period of establishment Ao gains, but Lp gains later on. In this type of experiment soil conditions and plants change systematically in the course of time and this is the reason why the course line is not straight after the 3 rd harvest in spite of the constant climatic conditions and regular clipping regime. 


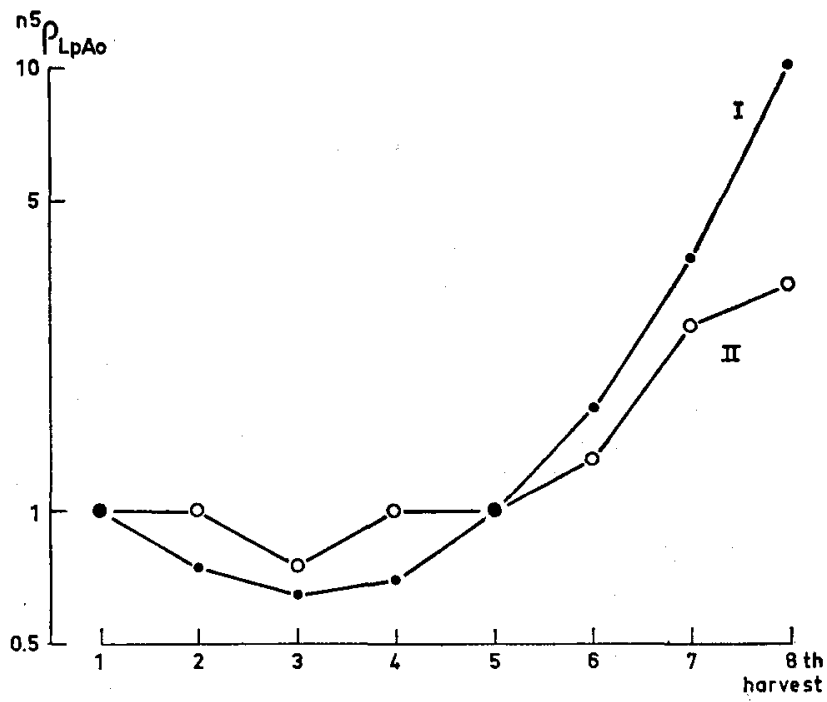

Fig. 6

Relative replacement rates presented as course lines for Lolium (Lp) with respect to Anthoxanthum (Ao) grown in pots, calculated from the dry-matter yields (I) and the number of sprouts after cutting (II). The experimental conditions are described in section 5 .

FIG. 7. Replacement diagrams based on the number of sprouts of Lolium (Lp) and Anthoxanthum (Ao) at eight successive harvests.
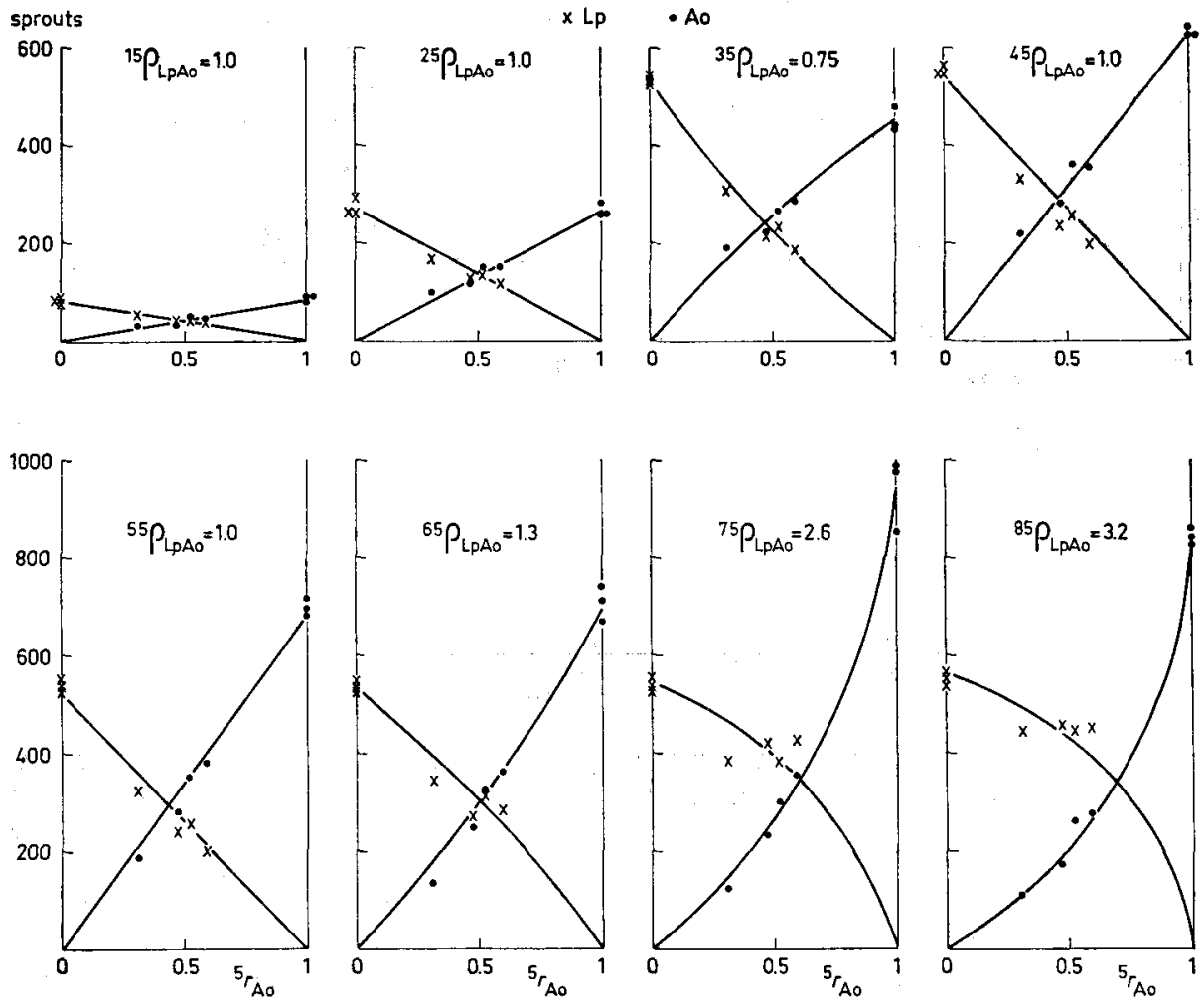

Neth. J. agric. Sci., Vol. 13 (1965) No. 2 (June) 
The number of sprouts is given in the replacement diagram of FIG. 7, again with the 5th harvest as a reference. Measured in this way the species are also exclusive. The RRR is presented by course line II in FIG. 6. This course line is similar to the one calculated from the dry-matter yields, but for somewhat smaller slopes. Apparently changes in number of sprouts lag behind changes in dry-matter yields. This is very pronounced in the last harvest. Here the amount of Ao in the mixture was so low that there was practically no material above the $4 \mathrm{~cm}$ level of clipping, but below that level some sprouts were left. The experiment came to an end when these also vanished after some weeks.

The difference between the course lines calculated in both ways is so large that it is not feasible to base the calculations on the number of sprouts of one harvest and on the herbage yield of another. On the other hand, this difference is so small that it is practically immaterial which method of measurement is adopted throughout an experiment.

The similarity between both course lines in FIG. 6 is not spurious as is shown in FIG. 8, in which the yields and the number of sprouts in successive harvests are

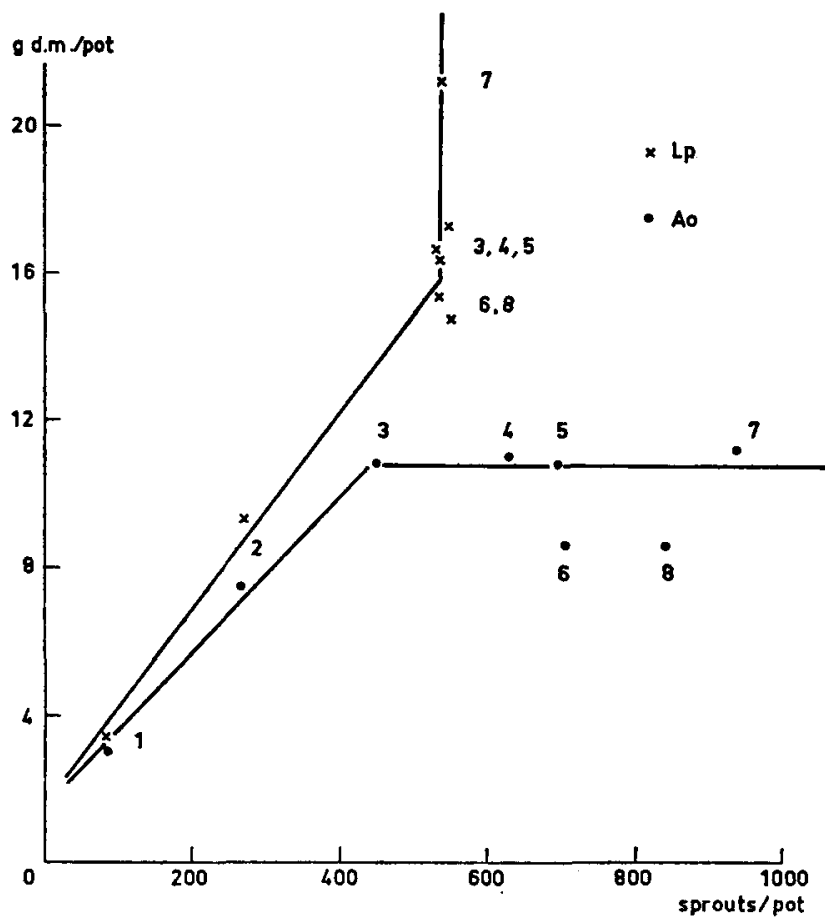

Fig. 8

Average dry-matter yields and average number of sprouts of the monocultures of Lolium (Lp) and Anthoxanthum (Ao) for the 8 harvests. The harvest number is given near the points (during the growth of the 7th harvest the lamps were left burning continuously for several days, causing high yields at the 7 th harvest).

plotted against each other for both species grown in monoculture. After establishment the yield of $L p$ varied but the number of sprouts remained the same, whereas the reverse was the case for Ao. However, the similarity of the course lines I based on dry-matter yields in FIG. 2 and 6, which are both calculated from FIG. 4 , is due to the maintenance of constant conditions and is absent in experiments with varying temperature, light and soil conditions (cf. section 2).

It is convenient to use the herbage yields in experiments harvested at frequent inter- 
vals, whereas counting sprouts is preferred in experiments harvested occasionally only. Of course the number of sprouts is the only practical measure if pastures are grazed. Many competition experiments with perennial grassland species are without the monocultures under natural conditions. In these cases it is only possible to calculate RRR on basis of observations in the mixture. As mentioned in section 2 , it is then difficult to conclude which species gains unless the experiments are of long duration or RRR differs widely from 1 . It is then practically impossible to study mutual interference of species within a season.

\section{RE F E R E N C ES}

Bergh, J. P. VAN DEN, and 1962 W. Th. Elberse

BERGH, J. P. VAN DEN, and 1960 C. T. DE WIT

ENNIK, G. C.

FISHER, R. A.

HARLAN, H. V., and M. L. Martini

LI, C. C.

WIT, C. T. DE

Competition between Lolium perenne L. and Anthoxanthum odoratum L. at two levels of phosphate and potash. J. Ecol. $50,87-95$.

Concurrentie tussen timothee (Phleum pratense L.) en reukgras (Anthoxanthum odoratum L.). Jaarb. I.B.S. 1960, Wageningen, $155-165$.

1960 De concurrentie tussen witte klaver en Engels raaigras bij verschillen in lichtintensiteit en vochtvoorziening. Jaarb. I.B.S. 1960. Wageningen, 37-50.

1930 The genetical theory of natural selection. New York.

1938 The effect of natural selection in a mixture of barley varieties. J. agric. Res. 57, 189-199.

1955 Population genetics. Chicago.

1960 On competition. Versl. Landbk. Onderz. No. 66.8, 82 pp.

1961 Space relationships within populations of one or more species. Soc. exp. Biol. Symp. 15, 314-329.

and G. C. ENNIK

1958 Over concurrentie. Jaarb. I.B.S. 1958. Wageningen, 59-73. 